\title{
The Future of Antibiotics: Emerging Technologies and Stewardship
}

Jeffrey L. Fortman ${ }^{1}$ and Aindrila Mukhopadhyay ${ }^{2, *}$

${ }^{1}$ American Association for the Advancement of Science, Science \& Technology Policy Fellow, Office of the Deputy Assistant Secretary of Defense for Chemical and Biological Defense, Washington, DC 20301, USA

${ }^{2}$ Biological Systems and Engineering, Lawrence Berkeley National Laboratory, Berkeley, CA 94720, USA

*Correspondence: amukhopadhyay@lbl.gov (A. Mukhopadhyay).

Keywords: antibiotics; policy; synthetic biology; natural products.

Antibiotic resistance is on the rise while the number of antibiotics being brought to market continues to drop. While this is a dire situation, a number of emerging technologies have the potential to reverse this trend. These, and supporting legislative initiatives, promise to stave off the post-antibiotic era.

\section{A Need for New Antibiotics}

Antibiotics are among the most important discoveries of the 20th century and the most important class of pharmaceuticals. For a few dollars, antibiotics can cure a potentially fatal infection in a young and otherwise healthy person, enabling decades of productive life. In addition, antibiotics facilitate our ability to conduct all manner of invasive modern medicine. Without antibiotics, organ transplants, joint replacements, and routine surgeries would be nearly impossible due to the threat of postoperative infection. Unfortunately, inappropriate use and natural selection have led to a rise in antibiotic resistance in most of the world's common pathogens. This, combined with poor economic incentives to develop new antibiotics, has resulted in an increase in deaths from infection and a decrease in new antibiotic development. A report commissioned by the United Kingdom government predicts that, if no steps are taken to combat antimicrobial resistance, the world will suffer an additional 10 million deaths every year by 2050 and a cumulative loss of $\$ 100$ trillion in productivity during that timei. This problem has been recognized and written about at the highest levels, culminating in a National Action Plan for the United States, issued by the White Housei.

The current system for antibiotic development and deployment presents many opportunities for revision, and there are a number of technological advances that show promise for augmenting the discovery and development of new antibiotics. Genomic, metagenomic, and functional genomic analyses of microbes, plants, and even marine invertebrates, coupled with synthetic biology techniques, promise to open the world's natural products at a rate that would outpace antibiotic discovery 
at its peak in the 1940s and 1950s when more than a dozen first-generation drugs were brought to market.

Beyond the scope of this paper there are biological therapies, such as phage [1] and antibodies [2], as well as vaccines [3] that are being developed to combat bacterial agents traditionally treated with antibiotics, and also completely novel approaches, such as DARPA's (Defense Advanced Research Projects Agency) bionic spleen [4], that provide new or alternative routes to treating infections. In addition to these new approaches, traditional synthetic organic and medicinal chemistry remain crucial tools for combatting antimicrobial resistance. These technologies, combined with a strong commitment to antibiotic stewardship, are our best chance to keep the human race on the winning side in the war against infection for the foreseeable future.

\section{Genomic and Metagenomic Mining}

Since the discovery of penicillin, natural products have been the major source of antibiotics. However, existing policies, the rate of emerging antibiotic resistance, and the economic burden of drug development have made it challenging for major pharmaceutical companies to continue their natural product screening and discovery programs; at the same time, drug development is beyond the means of academic researchers. Thus, even newly discovered natural products with antibiotic potential may be left undeveloped unless exceptional promise can be demonstrated. Technologies are emerging that will allow us to better assess and utilize what many believe to be the undiscovered majority of natural products and bring new life to the fields of natural products and, by extension, antibiotics.

A group of small companies, such as Ecopia BioSciences, Warp Drive Bio, and Radiant Genomics among others, have been changing this trend. In 2003, Ecopia BioSciences, Inc. published a strategy that utilized a bioinformatic approach to drug discovery [5]. They were able to identify novel compounds using predictive analysis of clusters of small-molecule biosynthesis genes. They confirmed and expanded on the work of others who found that there were often many small-molecule biosynthesis pathways per organism in many of the natural product-rich genera, and that most of these did not express these pathways under standard laboratory culture conditions. This previously unexplored space represents tremendous chemical diversity. Gene sequence-based analyses to predict the structure of the associated metabolites is being refined and augmented by Warp Drive Bio and others in order to improve both discovery and development of microbial natural products. Functional metagenomic expression has been used for many years by academics, such as Jo Handelsmann and John Clardy, to discover antimicrobial compounds. Now companies, such as Radiant Genomics, are using a combination of bioinformatics, heterologous expression, and host engineering to harness the vast biosynthetic potential of nature that has been heretofore invisible to natural product researchers. These culture-independent methods of pathway discovery provide a route to access the vast majority of nature's undiscovered pharmacopeia. While the process of taking a metagenomic, in silico, pathway discovery to a 
compound is still in its infancy, the authors feel that it will be a mature process in less than a decade.

University and National Laboratories are also working to move this technology forward. The Integrated Microbial Genomics Atlas of Biosynthetic gene Clusters (IMG-ABC) database hosted by the Department of Energy's Joint Genome Institute contains over 750000 small-molecule biosynthesis pathways collected from both whole-genome sequencing and metagenomic efforts [6]. If this effort is ultimately combined with the massive microbiome sequencing and functional genomics initiatives recently proposed [7], the potential impact of this approach should increase by an order of magnitude or more. In addition to sequence-based discovery, traditional methods and novel modifications thereof continue to reveal new antibiotic leads. A recent publication of the discovery of teixobactin by a group using innovative means to culture 'unculturable' microbes clearly illustrates the validity of this approach [8].

For the production of newly identified molecules, traditional methods for strain optimization have historically taken a decade or more to yield commercially relevant productivities. There are now a number of companies using synthetic biology techniques to shorten this timeline to months or a few years. There are also academics working on these types of approaches, but the fact that there are companies developing these technologies suggests that a commercially viable maturity point has been reached. This is highlighted by recent venture capital investments in startups such as Ginkgo Bioworks and Zymergen.

\section{Mapping the Microbiome}

Where the mapping and functional studies of the global microbiome will play an important role in natural product discovery, the mapping of the human microbiome will have a specific value in countering antibiotic resistance. It is becoming increasingly apparent that our personal microbiomes play a critical role in our physiology. While still nascent, our understanding of the interplay between our microbiome and our own cells is slowly coming into focus. The National Institutes of Health's Human Microbiome Project, started in 2008, is dedicated to accelerating precisely this understanding and represents a valuable effort that should be continued in the future. As we build our understanding of our bodies as ecosystems, we will be able to better target pathogens while leaving our native flora untouched, and rebuild a healthy community after finishing a course of broad-spectrum antibiotics.

\section{Diagnostics}

While outside of the scope of synthetic-biology-enabled drug discovery and development, the importance of diagnostics is too great to leave unmentioned. The requirement for strain-level identification needed for narrow-spectrum therapies, such as antibodies and bacteriophage, highlights the monumental value of diagnostics in improving our antibiotic stewardship. Rapid identification of a pathogen as either bacterial or viral, and microbial resistance or susceptibility to 
common therapeutic agents, will facilitate the prescription of the correct drug for a given infection. However, the majority of current diagnostics are growth-based. Growth-based assays often require days to determine the identity of a pathogen,

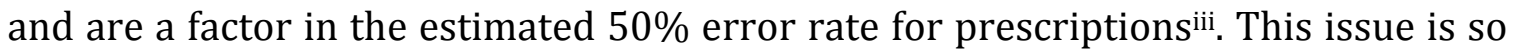
important that diagnostic development is one of the five Goals of the White House's National Action Plan for Combating Antibiotic-Resistant Bacteriaii.

\section{Stewardship}

The importance of antibiotic stewardship cannot be underestimated. While a number of excellent technologies are emerging, it should be recognized that the pool of physiological targets for antimicrobial action is finite. Thus, we as a society must not fail to improve our efforts surrounding antimicrobial stewardship, or we will find ourselves commonly facing microbial infections with no cures.

The issue of stewardship has two parallel areas to address, namely, their use in humans and their use in agriculture. As discussed above, we have an opportunity to use new tools and diagnostics to refine antibiotic prescriptions to humans and extend the efficacy of these compounds. The Centers for Disease Control and Prevention estimates the number of improper antibiotic prescriptions in the United States to be as high as $50 \%$ iii. Better diagnostics and additional education of both patients and physicians are both receiving attention in an effort to lower this number. Agricultural applications represent $75-80 \%$ of all antibiotic use in the United States [9] and thus are a major aspect of this challenge. While this has been recognized in the White House documents, it remains to be seen how the recommendations therein will be implemented. In order to protect the human pharmacopeia, while ensuring the food supply, it would be prudent to establish federal or international research programs focused on curtailing the use of antibiotics, that have corollaries in use in humans, from agricultural applications.

\section{Concluding Remarks}

The rise of resistance and the paucity of new antibiotics combine to paint a bleak picture of the future. However, new tools for discovering and developing naturalproduct-based antibiotics exist in various stages of development and are maturing rapidly. Economically, antibiotics - as a field - have been negatively impacted by their own success. The first generations were relatively easy to discover and bring to market. The pool of therapeutic targets has diminished while the standards for FDA approval have grown. This translates into a greatly increased cost of development. To counter this, the United States government has issued a number of reports and policy changes (Box 1). Similar initiatives are also being discussed, promoted, and implemented around the world. This confluence of emerging technology and pragmatic policy gives the authors tremendous optimism that the current trends toward a 'post-antibiotic era' can be reversed.

\section{Acknowledgments}

JLF is supported by a US Department of Defense (DOD) American Association for the Advancement of Science (AAAS) Fellowship, administered by Oak Ridge Institute for 
Science and Education (ORISE) through an interagency agreement between DOD and the U.S. Department of Energy (DOE). ORISE is managed by ORAU under DOE contract number DE-AC05-060R23100. AM is supported through contract DE-AC0205CH11231 between Lawrence Berkeley National Laboratory and the US Department of Energy, Office of Science, Office of Biological and Environmental Research. The United States Government retains and the publisher, by accepting the article for publication, acknowledges that the United States Government retains a non-exclusive, paid-up, irrevocable, world-wide license to publish or reproduce the published form of this manuscript, or allow others to do so, for United States Government purposes. All opinions expressed in this paper are the authors' opinions and do not necessarily reflect the policies and views of the DOD, DOE, or ORAU/ORISE.

\section{Disclaimer Statement}

JLF has financial interests in Lygos, Inc.

\section{Resources}

ihttp://amr-review.org/sites/default/files/AMR Review Paper - Tackling a crisis for the health and wealth of nations_1.pdf

iihttps://www.whitehouse.gov/sites/default/files/docs/national_action_plan_for_co mbating_antibotic-resistant_bacteria.pdf

iiihttps://www.whitehouse.gov/sites/default/files/microsites/ostp/PCAST/pcast_c arb_report_sept2014.pdf

\section{References}

1. Lu, T.K. and Koeris, M.S. (2011) The next generation of bacteriophage therapy. Curr. Opin Microbiol. 14, 524-531

2. DiGiandomenico, A. and Sellman, B.R. (2015) Antibacterial monoclonal antibodies: the next generation? Curr. Opin. Microbiol. 27, 78-85

3. Thanawastien, A. et al. (2015) Conjugate-like immunogens produced as protein capsular matrix vaccines. Proc. Natl. Acad. Sci. U.S.A. 112, E1143-E1151

4. Kang, J. H. et al. (2014) An extracorporeal blood-cleansing device for sepsis therapy. Nature Med. 20, 1211-1216

5. Zazopoulos, E. et al. (2003) A genomics-guided approach for discovering and expressing cryptic metabolic pathways. Nature Biotechnol. 21, 187-190

6. Hadjithomas, M. et al. (2015) IMG-ABC: A knowledge base to fuel discovery of biosynthetic gene clusters and novel secondary metabolites. mBio 6, e00932-15

7. Alivisatos, A.P. et al. (2015) A unified initiative to harness Earth's microbiomes. Science 350, 507-508 
8. Ling, L.L. et al. (2015) A new antibiotic kills pathogens without detectable resistance. Nature 517, 455-459

9. Hollis, A. and Ahmed, Z. (2013) Preserving antibiotics, rationally. N. Engl. J. Med. $369,2474-2476$

10. Clift, C. et al. (eds) (2015) Towards a New Global Business Model for Antibiotics Delinking Revenues from Sales. The Royal Institute of International Affairs, Chatham House

\section{Box 1. Policies for Promoting Antibiotic Development}

While there is much promising technology being moved forward to combat bacterial infections, the other, and perhaps more difficult, hurdle, is economic. The estimated cost of bringing a drug to market is between $\$ 500$ million and $\$ 2.5$ billion. The options for funding these efforts range from pure market-force models, where the maximum value of a human life is calculated and used to determine the price of treatment, to a fully socialized model where the government pays the entire cost of discovery, development, manufacturing, and distribution. The pragmatic answer lies between these two extremes. Models for delinking antibiotic revenues from sales have been studied, and they merit analysis and consideration well beyond the scope of this paper [10]. The Generating Antibiotic Incentives Now (GAIN) Act was passed in 2011 to incentivize the pharmaceutical industry to invest in this area. More recently, an effort to mitigate the cost of new antibiotic development, the Biomedical Advanced Research and Development Authority (BARDA), has recently used their other transactional authority to partner with AstraZeneca. This public-private partnership is an example of how public funds can be used to transition some of the risk of antibiotic development away from industry in order to make this area more attractive. 